\title{
Photopolymerization of Butyl Acrylate Microemulsion. Effect of Reaction Conditions and Additives on Fates of Desorbed Radicals
}

\author{
Ignác CAPEK
}

Polymer Institute, Slovak Academy of Sciences, Dúbravská cesta 9, 84236 Bratislava, Slovakia

(Received August 14, 1995)

\begin{abstract}
Oil/water microemulsion polymerizations of butyl acrylate photoinitiated by a UV light were investigated. The rate-conversion curve shows two rate intervals with the maximum rate $c a$. at $10-30 \%$ conversion and final conversion below $100 \%$. The rate of polymerization, particle size, final conversion and molecular weight were found to decrease with the addition of naphthalene, anthracene and benzophenone. The addition of acrylonitrile (a partly water-soluble monomer) increases the final conversion. The number of particles was found to increase in the presence of additives. The direct photolysis of the monomer swollen emulsifier micelles generates the initiating radicals. The retardation effects of additives are attributed to the transfer of energy and/or reactions of growing radicals with reaction intermediates.

KEY WORDS Microemulsion Photopolymerization / Micelle / Polymer Particles / Butyl Acrylate / Benzophenone / Aromatic Hydrocarbon /
\end{abstract}

Monomer/emulsifier microemulsions have been a subject of interest in colloid sciences for quite a long time. Such microemulsions are thermodynamically stable, optically isotropic, and transparent (translucent) liquid systems. They are homogeneous on a macroscopic scale but heterogeneous on a molecular scale. These systems are also described as globular micellar aggregates into which a monomer has been solubilized. Thus, they consist of oil and water domains separated by emulsifier monolayers.

Systematic phase behavior studies were first initiated by Winsor ${ }^{1}$ who showed that microemulsions, either optically transparent or translucent, were thermodynamically stable systems. These microemulsions can also exist in equilibrium with excess water, excess oil or both.

Microemulsion polymerizations offer convenient access to well-defined microlatex particles being typically one order of magnitude smaller than particles obtained by conventional emulsion polymerizations. There was no apparent constant rate period and no gel effect in studied thermally initiated microemulsion polymerizations. ${ }^{2-8}$ These microemulsions produced stable microlatexes with particle radii ranging from 20 to $50 \mathrm{~nm}$.

Generally, there are two methods for producing polymeric microemulsions. The first method is the thermally initiated polymerization of acrylate or methacrylate monomer using peroxodisulfate, $\alpha, \alpha^{\prime}$-azobisisobutyronitrile (AIBN) and dibenzoyl peroxide. ${ }^{2-8}$ The second polymerization procedure is photochemically induced polymerization in the presence of photosensitizer. ${ }^{9}$ The photochemical way appears a very promising to prepare polymers and polymer particles in microemulsion (direct and inverse)..$^{10-12}$

Earlier papers ${ }^{13,14}$ reported on the solution polymerization of acrylonitrile sensitized by aromatic hydrocarbons and benzophenone. The differences in efficiency of these aromatic hydrocarbons in photoinitiated polymerization was explained in terms of difference in ability to absorb the radiation used, self-quenching, monomer quenching, and hydrogen abstraction.

It seemed to us reasonable to study closely the kinetics of the microemulsion polymerization of butyl acrylate initiated by UV light in the presence of aromatic hydrocarbons and benzophenone. The presence and partitioning of additives (photoinitiator) between water and polymer particles (micelles) are expected to influence photophysical and photochemical events in the microdroplets and give information on radical formation. The hydrophobic butyl acrylate monomer is located in monomer swollen micelles where the initiating radicals are formed. The interactions of grade sodium dodecylsulfate (SDS)/butyl acrylate $\left(\mathrm{BA}^{*}\right)$ with additives in the ground or excited (with different energy of singlet and triplet) states are expected to influence the initiation mechanism (energy transfer, formation of intermediates, radicals, products). In order to evaluate this behavior, we follow the effects of additives on the kinetic, colloidal, and molecular weight parameters of the microemulsion polymerization and postpolymerization of butyl acrylate.

\section{EXPERIMENTAL}

\section{Materials}

Commercially available butyl acrylate (BA) and acrylonitrile (AN) monomers were purified by usual methods. The emulsifier used was the reagent-grade sodium dodecylsulfate (SDS) and analytical-grade naphthatalene (Nph), anthracene (Ant), benzophenone (BP), and butanol (BO1) (all from Fluka). SDS purity was checked by surface tension measurements to determine critical micellar concentration $(\mathrm{cmc})$. The latter gave a cmc value $\left(7.8 \mathrm{mmol} \cdot \mathrm{dm}^{-3}\right)$ in good agreement with literature values. Twice-distilled water was used as the polymerization medium.

\section{Polymerization Procedure}

Batch polymerizations were run at $23^{\circ} \mathrm{C}$ using the UV light of wavelength 313 or $365 \mathrm{~nm}$. In all runs the recipe comprises $100 \mathrm{~g}$ water and $10 \mathrm{~g} \mathrm{BA}$. Amounts of SDS, $\mathrm{Nph}$, Ant, BOL, and BP varied as shown later.

\section{Polymerization Techniques}

Polymerization experiments were performed on an 
optical bench using monochromatic light of the wavelength of $\lambda=323 \mathrm{~nm}$ or $365 \mathrm{~nm}$ at $23^{\circ} \mathrm{C}$. Equipment, measurement of radiation intensity and photopolymerization technique have been described elsewhere. ${ }^{13}$ Laser spectroscopy measurements have been described elsewhere. $^{15}$

\section{Polymer and Latex Characterization}

The polymerization techniques and measurements of particle size and number (a static and dynamic light scattering (LS)) were the same as described earlier. ${ }^{16,17}$ Conversion of monomer was determined by dilatometric measurements (checked by gravimetry). Limiting viscosity numbers were measured in acetone at $25^{\circ} \mathrm{C}$ and used to estimate viscosity-average molecular weights $\left([\eta]=6.85 \times 10^{-5} \cdot M_{v}{ }^{0.75}\right) .{ }^{18.19}$ An Ubbelohde viscometer was also used for measurement of the viscosity of the reaction mixture (microemulsion) at $25^{\circ} \mathrm{C}$. Flow times were converted to viscosity in $\mathrm{mPa} \cdot \mathrm{s}$ using the calibration curve constructed by measuring the flow times of solvents of known viscosities. Conductivity measurements of the microlatexes have been described elsewhere. ${ }^{20}$ The number-average molecular weights were measured on a Hewlett-Packard, Model 502, high speed membrane osmometer in toluene at $30^{\circ} \mathrm{C}$.

\section{RESULTS AND DISCUSSION}

\section{Microemulsion}

Microemulsions of BA, SDS, and water were found to be formed spontaneously. The optical transparency of microemulsion (SDS/BA) systems was higher ( $c a$. $86 \%$ ) than that of the (SDS/BA/Additive) systems. Polymeric microemulsions were less transparent than the monomeric ones, due to the larger particle size of polyeric dispersion. The number of micelles in the SDS/water system with $[\mathrm{SDS}]=0.54 \mathrm{~mol} \cdot \mathrm{dm}^{-3}$ (the average micelle diamer $D=4 \mathrm{~nm}$ and the emulsifier aggregation number $N_{\mathrm{a}}=66$ is ca. $5 \times 10^{21}$ per $\mathrm{dm}^{3} .^{21}$

Kinetic, Colloidal and Molecular Weight Parameters, Aromatic Hydrocarbons, and Benzophenone

Typical plots of the conversion curves for the microemulsion photopolymerization of butyl acrylate in the presence or the absence of additives are illustrated in Figure 1. The range of additive concentrations was selected so that it was possible to neglect the selfquenching. ${ }^{13}$ The linearity of conversion curves is observed over the low conversion range (up to $15-25 \%$ conversions for $\mathrm{Nph}, \mathrm{BP}, \mathrm{AN}$, and without additive or $12 \%$ for Ant).

Without any irradiation, no polymerization of BA takes place in the butyl acrylate microemulsion with or without additives, i.e., the rate of "dark" polymerization is zero. In separate experiments it was found that oxygen strongly inhibited the microemulsion photopolymerization of BA.

The conversion curves take on a shape more similar to that for "dead-end" polymerization. ${ }^{22}$ In all runs the final conversion is below $100 \%$ (Table I) and no gel effect appears.

Variation in the rate of microemulsion photopolymerization with conversion and the type of additive is expressed in Figure 2. Polymerization rates at different conversions (from Figure 1) were determined by nonlinear least squares regression analysis. This dependence shows two non-stationary distinct regions. In the first interval new particles are being created, the rate of primary particle formation reaches maximum, polymeriza-

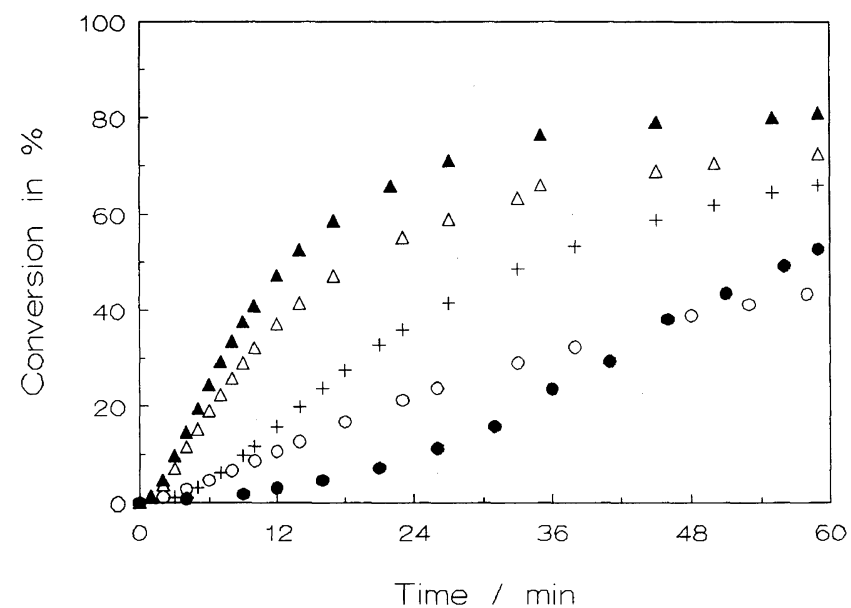

Figure 1. Variation of monomer conversion in the microemulsion polymerization of BA photoinitiated by UV light with the type of additive and reaction time. Recipe: $100 \mathrm{~g}$ water, $[\mathrm{BA}]=0.6 \mathrm{~mol} \cdot \mathrm{cm}^{-3}$, $[\mathrm{SDS}]=0.54 \mathrm{~mol} \cdot \mathrm{dm}^{-3}, \quad[\mathrm{Nph}]=3.86 \times 10^{-3} \mathrm{~mol} \cdot \mathrm{dm}^{-3}, \quad[\mathrm{Ant}]=$ $3.87 \times 10^{-5} \mathrm{~mol} \cdot \mathrm{dm}^{-3}, \quad[\mathrm{BP}]=3.74 \cdot 10^{-3} \mathrm{~mol} \cdot \mathrm{dm}^{-3}, \quad[\mathrm{AN}]_{3}=0.57$ $\mathrm{mol} \cdot \mathrm{dm}^{-3} \cdot \lambda=313 \mathrm{~nm}, I_{0}=1.1 \times 10^{-5}$ einstein $\cdot \mathrm{dm}^{-3} \cdot \mathrm{s}^{-1}$, (A) without additive, $(\triangle) \mathrm{Nph},(+) \mathrm{AN},(\bigcirc) \mathrm{BP},(\bigcirc)$ Ant.

Table I. Variation of the kinetic, colloidal, and molecular weight parameters in the microemulsion polymerization of BA initiated by UV light with different additives ${ }^{\mathrm{a}}$

\begin{tabular}{|c|c|c|c|c|c|c|c|c|c|c|c|}
\hline \multirow{2}{*}{ Additive } & \multicolumn{2}{|c|}{$\begin{array}{c}R_{\mathrm{p}, \max } \mathrm{f} / \mathrm{mol} \cdot \mathrm{dm}^{-3} \cdot \mathrm{s}^{-1} \\
\left(\times 10^{4}\right)\end{array}$} & \multicolumn{3}{|c|}{ Conversion $/ \%$} & \multirow{2}{*}{$\frac{D^{\mathrm{g}}}{\mathrm{nm}}$} & \multirow{2}{*}{$\begin{array}{c}N^{\mathrm{h}} \\
\left(\times 10^{-18}\right) / \\
\mathrm{dm}^{3} \\
\text { Final } \\
\text { conv. }\end{array}$} & \multirow{2}{*}{$\begin{array}{c}M_{v}{ }^{\mathrm{i}} \\
\left(\times 10^{-6}\right) \\
\text { Final }^{-6} \\
\text { conv. }\end{array}$} & \multirow{2}{*}{$\begin{array}{c}M_{n}{ }^{\mathrm{j}} \\
\left(\times 10^{-6}\right) \\
\text { Final }^{\mathrm{d}} \\
\text { conv. }^{\mathrm{d}}\end{array}$} & \multirow{2}{*}{$\begin{array}{c}\eta^{\mathrm{k}} \\
\mathrm{mPs} \\
\text { Final } \\
\text { conv. }^{\mathrm{d}}\end{array}$} & \multirow{2}{*}{$\begin{array}{c}\kappa^{1} \\
\mathrm{mS} \\
\text { Final }_{\text {conv. }}^{\mathrm{d}}\end{array}$} \\
\hline & & $\begin{array}{c}\text { With additive/ } \\
\text { without } \\
\text { additive }^{\mathrm{e}}\end{array}$ & At $R_{\mathrm{p}, \max }{ }^{\mathrm{b}}$ & At $4 h^{c}$ & $\begin{array}{l}\text { Final } \\
\text { conv. }^{d}\end{array}$ & & & & & & \\
\hline 0 & 6.5 & 1.0 & 10.0 & 80 & 85 & 46 & 1.75 & 2.8 & 6.0 & 2.4 & 20.5 \\
\hline $\mathrm{Nph}$ & 5.4 & 1.2 & 11.6 & 75 & 80 & 46 & 1.6 & 2.4 & 4.9 & & 20.5 \\
\hline Ant & 1.9 & 3.4 & 23.6 & 75 & 80 & 35 & 3.6 & 2.1 & 4.4 & 3.0 & 20.0 \\
\hline $\mathrm{BP}$ & 1.3 & 4.9 & 12.7 & 70 & 75 & 34 & 3.5 & 1.1 & 2.0 & 4.2 & 20.5 \\
\hline
\end{tabular}

${ }^{\mathrm{a}}[\mathrm{BA}]=0.6 \mathrm{~mol} \cdot \mathrm{dm}^{-3},[\mathrm{SDS}]=0.54 \mathrm{~mol} \cdot \mathrm{dm}^{-3}, \lambda=313 \mathrm{~nm},[\mathrm{Nph}]=3.86 \times 10^{-3} \mathrm{~mol} \cdot \mathrm{dm}^{-3},[\mathrm{Ant}]=3.87 \times 10^{-3} \mathrm{~mol} \cdot \mathrm{dm}{ }^{-5},[\mathrm{BP}]=3.74 \times$ $10^{-3} \mathrm{~mol} \cdot \mathrm{dm}^{-3}, I_{0}=1.1 \times 10^{-5}$ einstein $\cdot \mathrm{dm}^{-3} \mathrm{~s}^{-1} \cdot{ }^{\mathrm{b}}$ Conversion at the $R_{\mathrm{p}, \max }{ }^{\mathrm{c}}$ Conversion at $4 \mathrm{~h}$. ${ }^{\mathrm{d}}$ Final conversion. ${ }^{\mathrm{e}}$ Ratios of the rates without and with additive. ${ }^{\mathrm{f}}$ Maximum rate of polymerization. ${ }^{\mathrm{g}}$ Particle diameter. ${ }^{\mathrm{h}}$ Number of particles. ${ }^{\mathrm{i}}$ Viscosity-average molecular weight. ${ }^{\mathrm{j}}$ Number-average molecular weight. ${ }^{\mathrm{k}}$ Specific viscosity. ${ }^{1}$ Conductivity. 
tion proceeds under monomer saturated conditions, and monomer-swollen micelles act as emulsifier and monomer reservoirs, and therefore the rate increases. ${ }^{23,24}$

For acrylonitrile polymerization photosensitized by $\mathrm{Nph}$, Ant, or BP the rate of polymerization was found to depend on the square root of hydrocarbon concentration (rate increased in the presence of these additives). ${ }^{13,14}$ In the present system, however, the addition of $\mathrm{Nph}$ or Ant decreased the rate of polymerization and the decrease was more pronounced with Ant. Both Nph and Ant absorb light and form excited singlet and triplet states (quantum yield of intersystem crossing is ca. $0.7-0.8$ ). Because of the different molar decadic extinction coefficients of aromatic hydrocarbons or ketones at $313 \mathrm{~nm}$ (see Table II) constant absorbance of the system swas achieved by varying the concentration of additive so that $A=\varepsilon$ [Additive], $l=$ constant, where $A$ is absorbance, $\varepsilon$ is the molar decadic extinction coefficient of additives, [additive] is its concentration in $\mathrm{mol} \cdot \mathrm{dm}^{-3}$, and $l$ is the path length in centimeters. It was seen that the concentrations of $\mathrm{Nph}$, Ant, and BP were in the concentration range where self-quenching did not affect the rate of polymerization. ${ }^{13}$

The dependence of the rate of BA polymerization on conversion is described by a curve with a maximum corresponding to a certain conversion. The position of the rate maximum which is located in the conversion

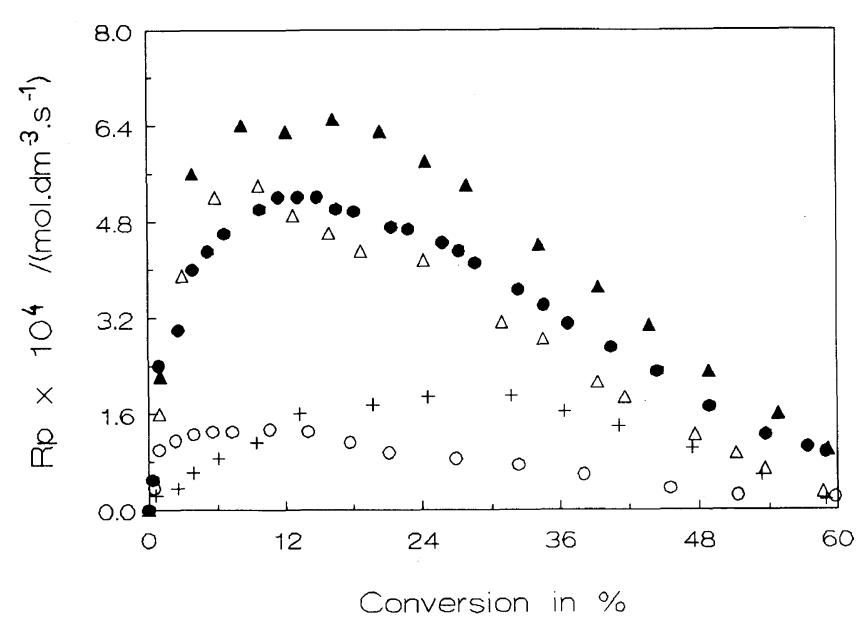

Figure 2. Variation of the rate of polymerization in the microemulsion polymerization of BA photoinitiated by UV light with the type of additive and conversion. $(\Delta)$ without additive, $(\triangle) \mathrm{Nph},(+)$ AN, (O) BP, (O) Ant. For other conditions, see legend to Figure 1. range from $10 \%$ to $25 \%$ seems to be inversely proportional to the rate of polymerization, i.e., the higher maximum rate the lower conversion at which it appears (Table I).

Table I shows that neither Nph (or Ant) nor BP act as a sensitizer even though the triplet activation energy varied from $68.6 \mathrm{kcal} \cdot \mathrm{dm}^{-3}$ for $\mathrm{BP}, 60.9 \mathrm{kcal} \cdot \mathrm{dm}^{-3}$ for $\mathrm{Nph}$ and to $42 \mathrm{kcal} \cdot \mathrm{mol}^{-1}$ for Ant. Thus the energy transfer from each additive to BA/SDS aggregates is not operative.

The observed decrease in the BA polymerization rate, thus, may be interpreted as a consequence of competitive reactions between $\mathrm{BA}^{*}$ or SDS/BA* (formation of exciplex and initiating radicals) on the one hand and energy transfer from $\mathrm{BA}^{*}$ or $\mathrm{SDS} / \mathrm{BA}^{*}$ to the ground state of additive molecules on the other, as well as a result of reactions between $\mathrm{BA}^{*}$ or SDS/BA* and additives. The radicals (from $\mathrm{BA}^{*}$ or $\mathrm{SDS} / \mathrm{BA}^{*}$ ) cam react with naphthanene (anthracene) in a reaction sequence as explanation of the inhibitory effect of aromatic hydrocarbon on thermally initiated polymerization of vinyl monomers. ${ }^{25}$ However, in the presence of naphthalene $\left(\mathrm{ca} .0 .001-0.01 \mathrm{~mol} \cdot \mathrm{dm}^{-3}\right)$ neither the rate of thermally initiated microemulsion polymerication of BA nor the molecular weight of poly(BA) decreased.

As can be seen from the results in Table I, benzophenone very strongly decreases the rate of polymerization as well as the molecular weight of poly(BA) formed. Similar behavior of BP was reported in the photopolymerization of $\mathrm{AN}$ initiated by $\mathrm{Nph}^{14}$ where the results are discussed in terms of reaction of propagating radicals with ketyl radicals. In the SDS/BA microemulsions BP in its triplet state decays according to several relaxation processes: 1) deactivation through a nonradiative process $(\mathrm{k}), 2)$ generation of a radical pair through hydrogen abstraction from the emulsifier $\left(\mathrm{k}_{\mathrm{r}}\right)$, and quenching by monomer $\left(\mathrm{k}_{\mathrm{q}}\right)$. The exit of a radical depends on the nature of the solute, emulsifier or co-emulsifier and is greatly favored by the location of $\mathrm{BP}$ very close to the water phase. ${ }^{26}$ Positive interaction $\mathrm{BP}^{*} / \mathrm{SDS}$ should be realized in the hydrophobic core of monomer swollen micelles. Thus, the sensitizer effect of BP which is interpreted in terms of hydrogen abstraction by ${ }^{3} \mathrm{BP} *$ from the $\mathrm{CH}_{2}$ backbone of SDS is not operative in the present system. Here, deactivation events such as quenching by monomer and self-quenching (see later) probably decrease the triplet state lifetime or $\left[{ }^{3} \mathrm{BP}^{*}\right]$.

Table II. Variation of the kinetic, colloidal, and molecular weight parameters in the microemulsion polymerization of BA initiated by UV light with different additives ${ }^{\text {a }}$

\begin{tabular}{|c|c|c|c|c|c|c|c|c|c|c|c|}
\hline \multirow{2}{*}{ Additive } & \multirow{2}{*}{$\frac{\varepsilon^{\mathrm{e}}}{\mathrm{dm}^{3} \cdot \mathrm{mol}^{-1} \cdot \mathrm{cm}^{-1}}$} & \multicolumn{2}{|c|}{ Conversion $/ \%$} & \multirow{2}{*}{$R^{\mathrm{f}}$} & \multicolumn{2}{|c|}{$\frac{D}{\mathrm{~nm}}$} & \multicolumn{3}{|c|}{$\begin{array}{c}N \\
\left(\times 10^{-18}\right) / \mathrm{dm}^{3}\end{array}$} & \multirow{2}{*}{ 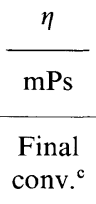 } & \multirow{2}{*}{$\begin{array}{c}\kappa \\
\mathrm{mS} \\
\text { Final } \\
\text { conv. }^{\mathrm{c}}\end{array}$} \\
\hline & & $\begin{array}{c}24 \% \\
\text { conv. }^{\mathrm{b}}\end{array}$ & $\begin{array}{l}\text { Final } \\
\text { conv. }^{\mathrm{c}}\end{array}$ & & $\begin{array}{l}24 \% \\
\text { conv. }\end{array}$ & $\begin{array}{l}\text { Final } \\
\text { conv. }^{\mathrm{c}}\end{array}$ & $\begin{array}{l}24 \% \\
\text { conv. }^{\mathrm{b}}\end{array}$ & $\begin{array}{l}\text { Final } \\
\text { conv. }^{\mathrm{c}}\end{array}$ & $\begin{array}{l}\text { Final conv./ } \\
24 \% \text { conv. }^{d}\end{array}$ & & \\
\hline 0 & - & 23 & 60 & 0.7 & 56 & 51 & 0.4 & 1.0 & 2.5 & 4.4 & 18 \\
\hline Ant & 12300 & 25 & 50 & 0.5 & 40 & 36 & 1.26 & 2.64 & 2.1 & 4.5 & 18 \\
\hline BP & 143 & 24 & 67 & 0.33 & 42 & 36 & 1.0 & 3.0 & 3.0 & 4.5 & 17 \\
\hline $\mathrm{AN}(2)$ & 0.12 & 24 & 41 & 0.8 & 44 & 41 & 0.9 & 1.6 & 1.8 & 3.5 & 18 \\
\hline
\end{tabular}

${ }^{\mathrm{a}} c f$. Table I. ${ }^{\mathrm{b}} 24 \%$ conversion (light was turned off). ${ }^{\mathrm{c}}$ Final conversion. ${ }^{\mathrm{d}}$ Ratio of the number of particles at final and $24 \%$ conversion. ${ }^{\mathrm{e}}$ Decadic molar extinction coefficient of hydrocarbons at $313 \mathrm{~nm} .{ }^{\mathrm{f}} R$, ratios of the rates at $27 \%$ conversion and $24 \%$ conversion. 
Table III. Variation of the kinetic, colloidal, and molecular weight parameters in the microemulsion polymerization of BA initiated by UV light with different additives ${ }^{\mathrm{a}}$

\begin{tabular}{|c|c|c|c|c|c|c|c|c|c|}
\hline \multirow{2}{*}{ Additive } & \multirow{2}{*}{$\frac{\begin{array}{c}R_{\mathrm{p}, \max }^{\mathrm{d}} \\
\left(\times 10^{4}\right)\end{array}}{\mathrm{mol} \cdot \mathrm{dm} \cdot \mathrm{s}^{-1}}$} & \multicolumn{2}{|c|}{ Conversion $/ \%$} & \multirow{2}{*}{$\frac{D^{\mathrm{e}}}{\mathrm{nm}}$} & \multirow{2}{*}{$\begin{array}{c}N^{\mathrm{f}} \\
\left(\times 10^{-18}\right) / \mathrm{dm}^{3} \\
\text { Final } \\
\text { conv. }{ }^{\mathrm{c}}\end{array}$} & \multirow{2}{*}{$\begin{array}{c}M_{v}^{\mathrm{g}} \\
\left(\times 10^{-6}\right) \\
\text { Final } \\
\text { conv. }{ }^{\mathrm{c}}\end{array}$} & \multirow{2}{*}{$\begin{array}{c}M_{n}^{\mathrm{g}} \\
\left(\times 10^{-6}\right) \\
\text { Final } \\
\text { conv. }\end{array}$} & \multirow{2}{*}{$\begin{array}{c}\eta^{\mathrm{i}} \\
\text { mPs } \\
\text { Final } \\
\text { conv. }^{\mathrm{c}}\end{array}$} & \multirow{2}{*}{$\begin{array}{c}\kappa^{\mathrm{j}} \\
\mathrm{mS} \\
\text { Final } \\
\text { conv. }\end{array}$} \\
\hline & & At $R_{\mathrm{p}, \max }{ }^{\mathrm{b}}$ & $\begin{array}{l}\text { Final } \\
\text { conv. }^{\mathrm{c}}\end{array}$ & & & & & & \\
\hline 0 & 6.2 & 12 & 78 & 48 & 1.4 & 2.2 & $?$ & 2.6 & 19 \\
\hline $\mathrm{Nph}$ & 6.2 & 25 & 84 & 46 & 1.6 & 2.4 & $?$ & 7.2 & 21 \\
\hline Ant & 2.2 & $20-50$ & 74 & 39 & 2.5 & 1.9 & $?$ & 3.0 & 20.5 \\
\hline
\end{tabular}

${ }^{\mathrm{a}} \lambda=365 \mathrm{~nm}, I_{0}=2.0 \times 10^{-5}$ einstein $\cdot \mathrm{dm}^{-3} \cdot \mathrm{s}^{-1} .{ }^{\mathrm{b}}$ Conversion at $R_{\mathrm{p}, \max }{ }^{\mathrm{c}}$ Final conversion $(\mathrm{ca}$. at $3 \mathrm{~h})$. ${ }^{\mathrm{d}} \mathrm{Maximum}$ rate of polymerization. ${ }^{\mathrm{e}}$ Particle diameter. ${ }^{\mathrm{f}}$ Number of particles. ${ }^{\mathrm{g}}$ Viscosity-average molecular weight. ${ }^{\mathrm{h}}$ Number-average molecular weight. ${ }^{\mathrm{i}}$ Specific viscosity. ${ }^{\mathrm{j}}$ Conductivity.

The results in Table III show that polymerization does not depend on $\mathrm{Nph}$ and is much slower with Ant and BP $(\lambda=365 \mathrm{~nm})$. Here Nph does not absorb light while Ant and BP do so. The decrease in molecular weight was observed with Ant and BP. These results indicate that the excited states (intermediates) of Ant or BP take part in the reaction.

Two transient species were reported to absorb light after the photolysis of BP-the triplet state (short-lived; below $1 \mu \mathrm{s}$ ) and the ketyl radical (long-lived; above $100 \mu \mathrm{s}) .{ }^{27}$ As a general rule, the reaction of ${ }^{3} \mathrm{BP}^{*}$ with hydrogen donors (reaction components) leads to the formation of a low amount of very reactive transferred radicals and high amount of much less reactive ketyl radicals. ${ }^{28}$ However, the ketyl radicals are known to act as a terminating agent of the growing radicals. No sensibilization effect of BP indicates that the ketyl radicals dominare the polymerization process. Thus, the initiating radicals formed by decomposition of excited (BA/SDS) complexes are deactivated by ketyl radicals. This is supported by the low rates of polymerization. Besides, BP did not influence the thermally initiated microemulsion polymerization of BA (rate of polymerization and the molecular weight). The decreased molecular weights in the photoruns thus may be attribued to the reactions of the triplet state ${ }^{3} \mathrm{BP}$ (diphenylhydroxymethyl (ketyl) radicals) with growing radicals. ${ }^{28}$

The kinetic curves of ${ }^{3} \mathrm{Ant} *$ photolysis were used to estimate the lifetime and first-order decay rate constant as a function of the reaction medium. The triplet lifetime and decay rate constant of Ant* were found to be $4.6 \mu \mathrm{s}$ in toluene and $56.2 \mu \mathrm{s}$ in the microemulsion and $2.18 \times 10^{5} \mathrm{~s}$ in toluene and $0.18 \times 10^{5} \mathrm{~s}$ in the microemulsion. This suggests that, owing to the compartmentalization of Ant, bimolecular reactions which take place in toleuene are avoided (or depressed) in micelles. The exit of hydrophobic Ant radicals is hindered which should increase the cage effect or deactivation events. Indeed, the addition of Ant led to decrease of the rate of polymerization. Thus, interactions of ${ }^{3} \mathrm{Ant}^{*}$ (radical intermediates) with growing or enterring radicals occur. In the homogeneous medium, however, the triplet state of Ant* was observed to be an efficient sensitizer for the styrene and AN photopolymerization. ${ }^{13,14,29}$

The trends of $R_{\mathrm{p}}$ indicate that the number of particles should be lower in the systems with additives. The experimental results show that the reverse is true. Thus, the rate of polymerization is inversely proportional to the number of particles. No definite statement is available

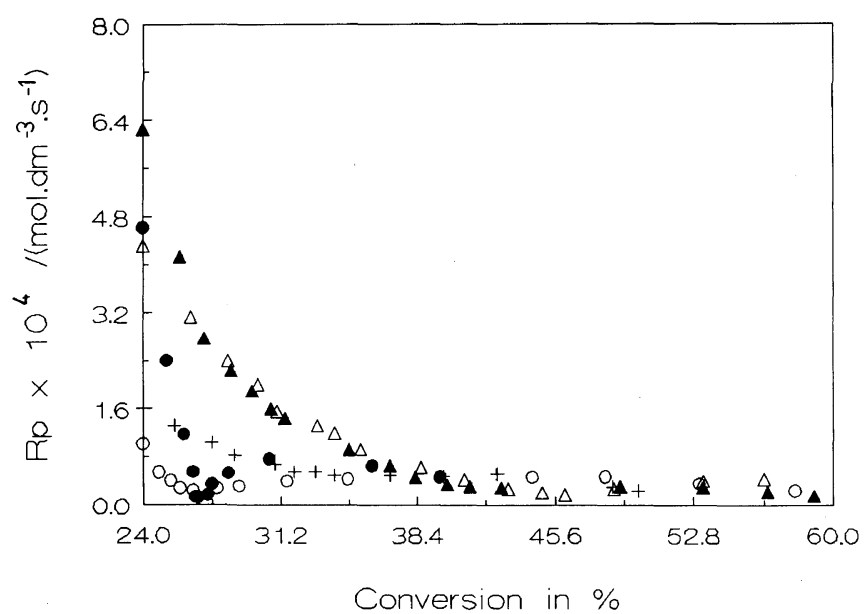

Figure 3. Variation of the rate of polymerization in the microemulsion polymerization of BA photoinitiated by UV light after illumination with the type of additive and conversion. ( $\boldsymbol{\Delta})$ without additive, $(\triangle)$ $\mathrm{Nph},(+) \mathrm{AN},(\bigcirc) \mathrm{BP},(\bigcirc)$ Ant. For other conditions, see legend to Figure 1.

yet to account for this effect. We suppose that the short stoppage of particle growth caused by the additive decreases the flux of monomer from micelles to particles which may favor the nucleation of additional micelles.

The strong decrease in $R_{\mathrm{p}}$ at high conversion may be discussed in terms of decrease of both the monomer concentration in the micelles or particles (reaction loci) and rate of initiation because the BA monomer itself acts as a radical precursor. ${ }^{15}$ The location of the monomer in the particle core decreases the absorption efficiency of butyl acrylate (light does not penetrate in polymer particles).

To evaluate the fate of radicals in the microemulsion polymerization of $\mathrm{BA}$, postpolymerization experiments were carried out (see Figure 3 and Table II). In all runs the rate of polymerization decreased with conversion. The abrupt decrease in $R_{\mathrm{p}}$ is observed shortly after the cessation of irradiation. The decrease in $R_{\mathrm{p}}$ is more pronounced with BP (see Table II, column R). Nph and Ant are water-insoluble components while BP is partly soluble in water. The exit of ${ }^{3} \mathrm{BP}^{*}$ (ketyl radicals) species from particles favors deactivation (reduction, hydroxylation, the water-phase termination). The decay curve indicates that the system contains two radicals: 1) fast decaying and 2) slow decaying radicals. The high reactive radicals which include primary radicals, growing (mobile) radicals and desorbed (monomeric) radicals disappears shortly after cessation of illumination. The more stable 
radicals responsible for polymerization at longer postpolymerization time include entangled (polymer matrix) or other resonance stabilized radicals. Thus, the concentration of mobile radicals decreases very fast with conversion while that of less reactive radicals decreases much less. The latter radicals are expected to govern the postpolymerization process.

The postpolymerization conversion interval (or the initiation efficiency of additives) was found to increase in the following order:

$$
\begin{gathered}
17 \%(\text { AN) }<25 \% \text { (Ant) }<37 \% \text { (without additive) } \\
<43 \%(\text { BP })<50 \%(\text { BOL })<56 \%(\mathrm{Nph})
\end{gathered}
$$

The addition of a high water-soluble monomer (AN) favors desorption and aqueous phase termination (concentration of radicals increases in water). The addition of a coemulsifier (BOL) increases the surface area of micelles which favors the re-entry of desorbed radicals (aqueous phase termination decreases).

Variation of the particle concentration with conversion and the type of additive shows that new particles are formed after cessation of illumination (Table II). Chain transfer to monomer or emulsifier in particles and desorption of monomeric radicals are assumed to favor nucleation. ${ }^{7,23,24}$ The much larger number of micelles than particles favors the re-entry of desorbed radicals and consequently the formation of new primary particles. This is the reason why the number of particles increases following polymerization where no further initiating radicals are produced.

Generally, viscosity is related to the microstructures of microemulsions. When below and above the percolation threshold, the viscosity of microemulsion varies slightly with monomer concentration. At the percolation threshold, viscosity steeply increases due to clustering of microdroplets. At a certain weight ratio monomer/polymer (conversion), thus, the percolation threshold is reached. The experimental results show that interparticle interactions may be also related to particle concentration.

By a combination of viscosity and conductivity measurements, viscosity can give some ideas about the structure of microemulsion. Conductivity is a measure of the mobility of charged carriers in the aqueous phase, while viscosity mirrors interactions between microdroplets. However, the conductivity was observed to be independent of additives and reaction conditions.

\section{Acrylonitrile}

The addition of the partly water-soluble acrylonitrile to $\mathrm{BA} / \mathrm{SDS}$ microemulsions increased both the watersoluble fraction of monomer and the desorption of radicals from particles. The increase in the hydrophilicity of a monomer is known to favor the contribution of the water-phase polymerization (homogeneous nucleation) and the desorption of monomeric (transferred) radicals.

The effects of $\mathrm{AN}$ concentration on the polymerization behavior are summarized in Figure 1 and Table IV. These results indicate that the addition of $\mathrm{AN}$ increases the final conversion, molecular weight, and number of particles and decreases the rate of polymerization at steady as well as at intermitent irradiation. The irradiation of the water-soluble fraction of AN and SDS (below and above CMC) does not proeuce the polymer product. This indicates than AN does not participate in the formation of the initiating radicals.

The decrease in $R_{\mathrm{p}}$ by the addition of AN may be attributed to increased desorption, slower water-phase polymerization and increased water-phase termination (the average time for termination increases, smaller polymers). ${ }^{20}$ The desorption rate constant for monomeric AN radicals is estimated to be $c a$. by $1-2$ orders in magnitude larger than that for BA ones. Desorption of radicals is known to disfavor growth events in particles and favor termination events in the aqueous phase and particles (re-entry of desorbed radicals). The fast decrease in $R_{\mathrm{p}}$ after the cessation of illumination favors the strong deactivation of radicals (the desorption of radicals, the water-phase termination, ... ). Essentially the same was observed with hydrophilic BP.

The increase of the final conversion in the presence of AN may be attributed to increased [BA] on the interface (AN serves as an extraction agent to decrease $\mathrm{BA}$ in polymer particles or increase BA on the particle surface). This may favor initiation and propagation events. The

Table IV. Variation of the kinetic, colloidal, and molecular weight parameters in the microemulsion

\begin{tabular}{|c|c|c|c|c|c|c|c|c|c|c|c|c|}
\hline \multirow[b]{2}{*}{$\mathrm{AN}$} & \multicolumn{2}{|c|}{$\begin{array}{c}R_{\mathrm{p}, \max }\left(\times 10^{4}\right) / \\
\mathrm{mol} \cdot \mathrm{dm}^{-3} \cdot \mathrm{s}\end{array}$} & \multicolumn{3}{|c|}{ Conversion $/ \%$} & \multicolumn{2}{|c|}{$D$} & \multirow{2}{*}{$\begin{array}{c}\begin{array}{c}N \\
\left(\times 10^{-18}\right)\end{array} \\
\mathrm{dm}^{3} \\
\begin{array}{c}\text { Final } \\
\text { conv. }\end{array}\end{array}$} & \multirow{2}{*}{$\begin{array}{c}M_{v} \\
\left(\times 10^{-6}\right) \\
\text { Final } \\
\text { conv. }^{d}\end{array}$} & \multirow{2}{*}{$\begin{array}{c}M_{n} \\
\left(\times 10^{-6}\right) \\
\text { Final }^{\text {conv. }}\end{array}$} & \multirow{2}{*}{$\begin{array}{c}\eta \\
\text { mPs } \\
\text { Final } \\
\text { conv. }\end{array}$} & \multirow{2}{*}{$\begin{array}{c}\kappa \\
\mathrm{mS} \\
\text { Final }^{\mathrm{d}} \\
\text { conv. }^{\mathrm{d}}\end{array}$} \\
\hline & & $\begin{array}{c}\text { With additive/ } \\
\text { without } \\
\text { additive }^{\mathrm{e}}\end{array}$ & At $R_{\mathrm{p}, \max }{ }^{\mathrm{b}}$ & At $4 h^{c}$ & $\begin{array}{l}\text { Final } \\
\text { conv. }\end{array}$ & $\begin{array}{l}24 \% \\
\text { conv. }\end{array}$ & $\begin{array}{l}\text { Final } \\
\text { conv. }\end{array}$ & & & & & \\
\hline \multicolumn{13}{|l|}{ 1) } \\
\hline 0.005 & 4.7 & 1.4 & 17.3 & 95 & 98 & & 42 & 2.25 & 3.3 & 7.2 & 2.6 & 21.5 \\
\hline 0.57 & 5.3 & 1.2 & 13.8 & 96 & 98 & & 40 & 2.6 & 2.9 & 6.1 & 3.2 & 21.5 \\
\hline \multicolumn{13}{|l|}{ 2) } \\
\hline 0 & 6.2 & 1.0 & 12 & 78 & - & 48 & - & 1.4 & 2.6 & 5.7 & 2.2 & 19 \\
\hline 0.05 & 6.2 & 1.0 & 13 & 97 & & 41 & & 2.4 & 2.7 & 5.9 & 2.5 & 21 \\
\hline 0.2 & 5.8 & 1.1 & 14 & 98 & & 39 & & 2.8 & 2.8 & 6.1 & 3.0 & 21 \\
\hline 0.5 & 5.2 & 1.2 & 19 & 97 & & 37 & & 3.3 & 2.9 & 6.3 & 3.3 & 21 \\
\hline
\end{tabular}
polymerization of BA initiated by UV light with AN concentration

${ }^{\mathrm{a}}[\mathrm{BA}]=0.6 \mathrm{~mol} \cdot \mathrm{dm}^{-3},[\mathrm{SDS}]=0.54 \mathrm{~mol} \cdot \mathrm{dm}^{-3}, \lambda=313 \mathrm{~nm}, I_{0}=1.1 \times 10^{-5}$ einstein $\cdot \mathrm{dm}^{-3} \cdot \mathrm{s}^{-1}$. ${ }^{\mathrm{b}}$ Conversion at the $R_{\mathrm{p}, \text { max }} \cdot{ }^{\mathrm{c}}$ Conversion at 4 h. ${ }^{d}$ Final conversion. ${ }^{\mathrm{e}}$ Ratios of the rates without and with additive. ${ }^{\mathrm{f}} 24 \%$ conversion (light was turned off). 
increase of viscosity with the [AN] is related to the interparticle interactions (agglomerations). At the high $[\mathrm{AN}]$ the agglomeration events are very operative. ${ }^{20}$

\section{The Effect of $p H$}

The effects of $\mathrm{pH}$ on the microemulsion polymerization of BA are summarized in Table V. The rate of polymerization is found to increase with $\mathrm{pH}$ and reach a maximum at $\mathrm{pH} 7$, and then decrease. The lower reactivity observed in the acidic media may be due to the protonation of emulsifier (the acid form), which may depress charge transfer events in the excited complex. In strongly alkaline media, the decrease is much more pronounced. Here, the negative surface charge of particles is supposed to suppress the percolation (exchange of reaction components including monomer and radicals between negatively charged polymer particles) which disfavors initiation and growth events.

Micelles and particles are very sensitive to small changes in the ionic strength of the aqueous solution and/or $\mathrm{pH}$. In SDS solution, the surface tension $(\gamma)$ decreases and hydrodynamic particle radius, $R_{\mathrm{h}}$, increases with $\mathrm{H}^{+}$concentration. With increasing $\mathrm{NaOH}$ $(\mathrm{pH}>9), R_{\mathrm{h}}$ decreases. The increased negative charge favors the compression of the diffuse double layer of micelles (particles). ${ }^{30}$ Indeed, the particle size of microlatex decreased from 48 to $39 \mathrm{~nm}$ in the $\mathrm{pH}$ range from 7 to 12 . Small particles were formed at very low $\mathrm{pH}$. This indicates that other factors influence polymerization, i.e., polymerization behavior is a complex function of surface charge density, aggregation number of emulsifier, and concentrations of monomer and initiating radicals.
Molecular weight, relative viscosity and conductivity were found to slightly vary with $\mathrm{pH}$.

\section{Butanol}

When adding alcohols there is positive attraction between the tails of emulsifier and alcohol. This positive effect leads to the formation of thermodynamically stable microemulsions with small particle size. The effects of butanol on the microemulsion polymerization of BA are summarized in Table VI. These results show that butanol decreases polymer particle size or increases the number of particles. The rate of polymerization is known to increase with particle number. However, the experimental results show that the reverse is true. The incorporation of butanol into microparticles is expected to change the attraction between SDS tails and BA* which is responsible for the formation of initiating radicals. In this case, the formation of radicals is suppressed as well as the growth events. The chain transfer to butanol leads to the formation of radicals with lower activity. Molecular weights were found to decrease with increasing butanol concentration.

The number of particles slightly varied with conversion under steady illumination and increased during postpolymerzation. This behavior differs from runs without butanol where the number of particles increased with conversion and particles were less nucleated after irradiation. ${ }^{15}$ This behavior may be attributed to the large surface area of BOL-microemulsions (co-emulsifier increases the surface area of micelles).

When polymerization is carried out at high butanol/SDS ratios, the size of latex particles is very large (cleassical emulsion is formed). This differs from the

Table V. Variation of the kinetic, colloidal, and molecular weight parameters in the microemulsion polymerization of BA initiated by UV light with $\mathrm{pH}$

\begin{tabular}{|c|c|c|c|c|c|c|c|c|}
\hline \multirow[b]{2}{*}{$\mathrm{pH}$} & \multirow{2}{*}{$\frac{R_{\mathrm{p}, \max }\left(\times 10^{4}\right)}{\mathrm{mol} \cdot \mathrm{dm}^{-3} \cdot \mathrm{s}^{-1}}$} & \multicolumn{2}{|c|}{ Conversion $/ \%$} & \multirow{2}{*}{$\begin{array}{c}D^{\mathrm{d}} \\
\begin{array}{c}\mathrm{nm} \\
\text { Final } \\
\text { conv. }^{\mathrm{c}}\end{array}\end{array}$} & \multirow{2}{*}{$\frac{\begin{array}{c}N^{\mathrm{e}} \\
\left(\times 10^{-18}\right)\end{array}}{\mathrm{dm}^{3}}$} & \multirow{2}{*}{$\begin{array}{c}M_{v}{ }^{\mathrm{f}} \\
\left(\times 10^{-6}\right) \\
\text { Final } \\
\text { conv. }^{\mathrm{c}}\end{array}$} & \multirow{2}{*}{$\begin{array}{c}\eta^{\mathrm{g}} \\
\mathrm{mPs} \\
\text { Final } \\
\text { conv. }^{\mathrm{c}}\end{array}$} & \multirow{2}{*}{$\begin{array}{c}\kappa^{\mathrm{h}} \\
\mathrm{mS} \\
\text { Final } \\
\text { conv. }\end{array}$} \\
\hline & & At $R_{\mathrm{p}, \max } \mathrm{b}$ & $\begin{array}{l}\text { Final } \\
\text { conv. }^{c}\end{array}$ & & & & & \\
\hline 2 & 3.6 & 20 & 89 & 41 & 2.3 & 2.3 & 2.2 & 21 \\
\hline 4 & 4.2 & 18 & 83 & 43 & 2.0 & 2.4 & 2.1 & 20 \\
\hline 7 & 6.2 & 12 & 78 & 48 & 1.4 & 2.6 & 2.2 & 19 \\
\hline 12 & 1.0 & 25 & 76 & 39 & 2.5 & 2.6 & 1.5 & 20.5 \\
\hline
\end{tabular}

${ }^{\mathrm{a}}[\mathrm{BA}]=0.6 \mathrm{~mol} \cdot \mathrm{dm}^{-3},[\mathrm{SDS}]=0.54 \mathrm{~mol} \cdot \mathrm{dm}^{-3}, \lambda=365 \mathrm{~nm}, I_{0}=2.0 \times 10$ einstein $\cdot \mathrm{dm}^{-3} \cdot \mathrm{s}^{-1} \cdot{ }^{\mathrm{b}}$ Conversion at $R_{\mathrm{p}, \max } \cdot{ }^{\mathrm{c}}$ Final conversion $(c a .3 \mathrm{~h})$. ${ }^{\mathrm{d}}$ Particle diameter. ${ }^{\mathrm{e}}$ Number of particles. ${ }^{\mathrm{f}}$ Viscosity-average molecular weight. ${ }^{\mathrm{g}}$ Specific viscosity. ${ }^{\mathrm{h}}$ Conductivity.

Table VI. Variation of kinetic, colloidal, and molecular weight parameters in the microemulsion polymerization of BA initiated by light with BOL concentration ${ }^{\mathrm{a}}$

\begin{tabular}{|c|c|c|c|c|c|c|c|c|c|}
\hline$[\mathrm{SDS}]$ & {$[\mathrm{BOI}$} & & Conver & $\mathrm{on} / \%$ & $D$ & $N$ & $M$ & $\eta$ & $\kappa$ \\
\hline $\mathrm{mol} \cdot \mathrm{dm}^{-3}$ & $\mathrm{~mol} \cdot \mathrm{dm}^{-3}$ & $\mathrm{~mol} \cdot \mathrm{dm}^{-3} \cdot \mathrm{s}^{-1}$ & At $R_{\mathrm{p}, \max }{ }^{\mathrm{b}}$ & $\begin{array}{l}\text { Final } \\
\text { conv. }\end{array}$ & $\begin{array}{l}\text { Final } \\
\text { conv. }\end{array}$ & $\begin{array}{l}\text { Final } \\
\text { conv. }^{\mathrm{c}}\end{array}$ & $\begin{array}{l}\text { Final } \\
\text { conv. }^{\mathrm{c}}\end{array}$ & $\begin{array}{l}\text { Final } \\
\text { conv. }^{c}\end{array}$ & $\begin{array}{l}\text { Final } \\
\text { conv. }\end{array}$ \\
\hline 0.54 & 0 & 6.2 & 12 & 78 & 48 & 1.4 & 2.6 & 2.2 & 19 \\
\hline 0.48 & 0.4 & 4.4 & 19 & 98 & 44 & 2.0 & 1.4 & 3.4 & 20.5 \\
\hline 0.43 & 0.4 & 4.4 & 18 & $70^{\mathrm{d}}$ & 37 & 3.0 & 1.72 & 3.3 & 21.5 \\
\hline 0.43 & 0.4 & 4.3 & 18 & $50^{\mathrm{e}}$ & 33 & 3.4 & 1.8 & - & - \\
\hline 0.37 & 1.0 & 1.8 & 18 & 94 & 375 & 0.003 & Emulsion & & \\
\hline
\end{tabular}

${ }^{\mathrm{a}} \lambda=365 \mathrm{~nm}, I_{0}=2.0 \times 10^{-5}$ einstein $\cdot \mathrm{dm}^{-3} \cdot \mathrm{s}^{-1} .{ }^{\mathrm{b}}$ At the $R_{\mathrm{p}, \max } \cdot{ }^{\mathrm{c}}$ Final conversion. ${ }^{\mathrm{d}}$ Postpolymerization; light turned off at $54 \%$ conversion. ${ }^{\mathrm{e}}$ Postpolymerization; light turned off at $36 \%$ conversion. 
microemulsion polymerization of styrene where stable polymeric microemulsions are formed. ${ }^{7}$

Increase in viscosity with [BOL] is a result of interparticle interactions between particles and microdroplets.

\section{CONCLUSION}

It follows from the foregoing that the photopolymerization of BA microemulsions forms stable and bluish-translucent microlatexes. The addition of aromatic hydrocarbons, benzophenone, acrylonitrile, and butanol increases the final particle number.

A common feature of BA polymerizations is the two-rate interval process. The rate of polymerization decreases with the addition of additives. The final conversion was found below $90 \%$ with and without additives. In the presence of acrylonitile and BOL, the final conversion increased and was close to $100 \%$ conversion. These results are discussed in terms of the desorption of monomeric (transferred) radicals, and water-phase termination and re-entry of monomeric radicals. The addition of additives was found to increase nucleation, i.e., the number of particles increased. Interparticle interactions were favored by the addition of acrylonitrile and butanol.

The postopolymerization conversion interval increases by BP and Nph and decreases by Ant, especially by AN. This behavior is discussed in terms of variation in the desorption rate, water-phase termination, re-entry rate and reaction of initiating or growing radicals with radicals generated during polymerization.

\section{REFERENCES}

1. P. A. Winsor, "Solvents Properties of Amphiphilic Compounds," Butterworths, London, 1954.

2. M. Antonietti, S. Lohmann, and C. V. Niel, Macromolecules, 25, 1139 (1992)

3. J. Jayakrishnan and D. O. Shah, J. Polym. Sci., Polym. Lett.
Ed., 22, 31 (1984)

4. M. T. Carver, U. Dreyer, R. Knoesel, F. Candau, and R. M. Fitch, J. Polym. Sci., A, Polym. Chem., 27, 2161 (1989).

5. A. P. Full, J. E. Puig, L. U. Gron, E. W. Kaler, J. R. Minter, T. H. Mourey, and J. Texter, Macromolecules, 25, 5157 (1992).

6. J. E. Puig, V. H. Perez-Luna, M. Perez-Gonzalez, E. R. Macias, B. E. Rodringuez, and E. W. Kaler, Colloid Polym. Sci., 271, 114 (1993).

7. J. S. Guo, E. D. Sudol, J. W. Vanderhoff, and M. S. Elaasser, $J$. Polym. Sci., A, Polym. Chem., 30, 691 (1992), ibid., 30, 703 (1992).

8. P. Potisk and I. Capek, Angew. Makromol. Chem., 222, 125 (1994).

9. C. Larpent and T. F. Tandros, Colloid Polym. Sci., 269, 1171 (1991).

10. M. T. Carver, U. Dreyer, R. Knoesel, F. Candau, and R. M Fitch, J. Polym. Sci., A, Polym. Chem., 27, 2161 (1989)

11. J. P. Fouassier, D. J. Lougnot, and I. Zuchowicz, Eur. Polym. J., 22, 933 (1986)

12. W. Reed, L. Guterman, P. Tundo, and J. H. Fendler, J. Am. Chem. Soc., 106, 1897 (1984).

13. J. Barton̆, I. Capek, and P. Hrdlovič, J. Polym. Sci., Polym. Chem. Ed., 13, 2671 (1975).

14. I. Capek and J. Bartoň, J. Polym. Sci., Polym. Chem. Ed., 13, 2691 (1975).

15. I. Capek and J. P. Fouassier, Eur. Polym. J., in press.

16. I. Capek, Makromol. Chem., 190, 789 (1989).

17. I. Capek and W. Funke, Makromol. Chem., 191, 2549 (1990).

18. I. Capek, J. Bartoň, and E. Orolinová, Chem. Zvesti, 38, 803 (1984).

19. J. Brandrup and E. H. Immergut, "Polymer Handbook," 3rd ed., John Wiley and Sons, Inc., New York, N.Y., 1989.

20. I. Capek and J. Batoň, Makromol. Chem., 186, 1297 (1985).

21. D. G. Marangoni, A. P. Rodenmiser, J. M. Thomas, and J. C. Kvak, Langmuir, 9, 438 (1993)

22. A. V. Tobolsky, J. Am. Chem. Soc., 80, 5927 (1958).

23. I. Capek and P. Potisk, Makromol. Chem. Phys., 196, 723 (1995)

24. I. Capek and P. Potisk, J. Polym. Sci., 33, 1675 (1995).

25. C. S. Bagdasaryan, "Theory of Radical Polymerization," Izdat Nauka, Moscow, 1966, p 169.

26. J. C. Scaiano, E. B. Abuin, and L. C. Steward, J. Am. Chem. Soc., 104, 5673 (1982).

27. J. P. Fouassier and D. J. Lougnot, J. Appl. Polym. Sci., 32, 6209 (1986).

28. J. Hutchison, M. C. Lambert, and A. Ledwith, Polymer, 14, 250 (1973).

29. R. G. W. Norish and J. P. Simons, Proc. Roy. Soc. (London), Ser., A, 251, 4 (1959).

30. W. Brown and J. Zhao, Langmuir, 10, 3395 (1994). 